\title{
Less invasive indicators of changes in thermodilution cardiac output by ventilatory changes after cardiac surgery Rose-Marieke B.G.E. Breukers ${ }^{a, b}$, Jore H. Willems ${ }^{a}$, Rob de Wilde ${ }^{b}$, Jos R.C. Jansen ${ }^{b}$ and A.B. Johan Groeneveld ${ }^{a}$
}

\begin{abstract}
Background and objective We studied whether changes in less invasive, noncalibrated pulse-contour cardiac output (by modified ModelFlow, COmf) and derived stroke volume variations (SVV), as well as systolic and pulse pressure variations, predict changes in bolus thermodilution cardiac output (COtd), evoked by continuous and cyclic increases in intrathoracic pressure by increases in positive endexpiratory pressure (PEEP) and tidal volume $\left(V_{t}\right)$, respectively.
\end{abstract}

Methods Prospective study on 17 critically ill postcardiac surgery patients on full mechanical ventilatory support, in the intensive care unit.

Results In contrast to systolic pressure variation and pulse pressure variation, SVV increased from (mean \pm SD) $6.2 \pm 4.4$ to $8.1 \pm 5.6$ at PEEP $10 \mathrm{cmH}_{2} \mathrm{O}(P=0.064)$ and to $7.8 \pm 3.5 \%$ at PEEP $15 \mathrm{cmH}_{2} \mathrm{O}(P=0.031)$, concomitantly with a $12 \pm 7$ and $11 \pm 8 \%$ decrease in COmf and COtd $(P<0.001)$, respectively. For pooled data, changes in COmf correlated with those in COtd $(r=0.55, P=0.002)$, but changes in SVV did not. Variables did not change when $V_{\mathrm{t}}$ was increased up to $50 \%$.

\section{Introduction}

In mechanically ventilated patients after cardiac surgery, cardiac filling pressures are insufficient predictors of preload-dependent cardiac output (CO), because, among other reasons, measurements are affected by transmitted airway pressure [1-10]. In contrast, left-sided dynamic indices, including stroke volume variations (SVV) and systolic and pulse pressure variations (SPV, PPV), which can be derived from the arterial pressure curve by pulsecontour analyses and are evoked, at least in part, by ventilation-induced alterations in cardiac loading conditions, may better predict preload responsiveness of CO than filling pressures [1-15]. Nevertheless, the value of dynamic indices also depends on ventilatory settings and thoracic compliance $[1,4,8,12,13,15]$. During ventilatory changes such as increases in positive end-expiratory pressure (PEEP) and tidal volume $\left(V_{\mathrm{t}}\right)$, right ventricular preload may decrease, afterload may increase and left ventricular preload, afterload and thereby $\mathrm{CO}$ may decrease [16]. Indeed, PEEP may increase left-sided dynamic indices, as studied in animals $[17,18]$ and humans $[12,14]$. Also, a low $V_{\mathrm{t}}$, in the course of lung-protective ventilation for instance, may decrease the predictive value
Conclusion A fall in COmf is more sensitive than a rise in SVV, which is more sensitive than systolic pressure variation and pulse pressure variation, in tracking a fall in cotd during continuous (and not cyclic) increases in intrathoracic pressure, in mechanically ventilated patients after cardiac surgery. This suggests a reduction in biventricular preload as the main factor in decreasing cardiac output and increasing SVV with PEEP. Eur J Anaesthesiol 26:863-867 (c) 2009 European Society of Anaesthesiology.

European Journal of Anaesthesiology 2009, 26:863-867

Keywords: cardiac surgery, ModelFlow, positive end-expiratory pressure, pulse-contour cardiac output, stroke volume variation

antensive Care, Institute for Cardiovascular Research, VU University Medical
Center, Amsterdam and ${ }^{b}$ Department of Intensive Care, University Hospital Leiden, Leiden, The Netherlands

Correspondence to A.B. Johan Groeneveld, MD, PhD, FCCP, FCCM, Department of Intensive Care Unit, VU University Medical Center, De Boelelaan 1117, $1081 \mathrm{HV}$ Amsterdam, The Netherlands

Tel: +31 20 4444178; fax: +3120 4442392; e-mail: johan.groeneveld@vumc.nl

Received 17 December 2008 Revised 17 February 2009 Accepted 19 February 2009

of dynamic indices for preload responsiveness as compared with high $V_{\mathrm{t}}$. The latter may decrease right ventricular preload, increase afterload and thereby affect left ventricular loading, stroke volume and arterial pressure variability $[4,8,12,13,15,19]$. In any case, SVV proved more sensitive to left ventricular preload than to afterloadinduced changes in $\mathrm{CO}$, may partly reflect invasive stroke volume variability and may thereby contribute to (even more easily obtained) SPV and PPV $[1,4,6,11,12,20]$. One method to measure SVV is by the modified ModelFlow pulse-contour technique that also allows evaluation of the course of CO less invasively, even without prior calibration. It performs well after cardiac surgery compared with thermodilution [21,22].

We evaluated whether noncalibrated, modified ModelFlow CO and SVV, as well as SPV and PPV, track thermodilution $\mathrm{CO}$ changes, used as a reference standard, during altered ventilatory settings in mechanically ventilated patients after cardiac surgery. Responses to continuous and cyclic intrathoracic pressures by stepwise increases in PEEP and $V_{\mathrm{t}}$, respectively, were compared to help explain the changes observed with PEEP. 


\section{Patients and methods Patients}

Seventeen patients (three female and 14 male patients) were included in the study after written informed consent was obtained. All patients underwent cardiac surgery, 11 coronary artery bypass grafting, five aortic valve replacements and one mitral valve repair. Postoperatively, the patients were admitted into the intensive care unit. In the operating room, they were already instrumented with a thermodilution pulmonary artery catheter $(8.5-\mathrm{Fr}$, CCO/VIP, Edwards Life Sciences, Santa Ana, California, USA) and a radial artery catheter. On arrival in the intensive care unit, patients were sedated with propofol $\left(1 \mathrm{mg} \mathrm{kg}^{-1} \mathrm{~h}^{-1}\right)$ or midazolam $\left(0.1 \mathrm{mg} \mathrm{kg}^{-1} \mathrm{~h}^{-1}\right)$ and sufentanil $\left(0.5 \mu \mathrm{g} \mathrm{kg}^{-1} \mathrm{~h}^{-1}\right)$. Patients were allowed to stabilize for 30-60 min. Patients' lungs were mechanically ventilated by volume-controlled ventilation with $V_{\mathrm{t}} \mathrm{s}$ of $10 \mathrm{ml} \mathrm{kg}^{-1}$ and a PEEP of $5 \mathrm{cmH}_{2} \mathrm{O}$. Ventilation frequency was set between 10 and 13 breaths $\min ^{-1}$ to maintain the arterial $P_{C O}$ in the normal range $(4.5-6.0 \mathrm{kPa})$. To maintain a steady state, nursing activities were minimized during the study.

\section{Modified ModelFlow method}

As described before [21-23], the ModelFlow method simulates ventriculoarterial coupling according to a three-element Windkessel model. The model has three principal components: aortic characteristic impedance, which represents the opposition of the aorta to pulsatile inflow; Windkessel compliance, which represents the ability of the arterial system to elastically store stroke output of the left ventricle; and peripheral resistance. The impedance and compliance of the model depend on pressure and total systemic peripheral resistance depends on many factors, including circulatory filling, metabolism, sympathetic tone and vasoactive drugs. The aortic characteristic impedance increases and the aortic Windkessel compliance decreases when aortic pressure increases. The nonlinear behaviour of the aortic wall could be a major source of error if not taken into account. The nonlinear relationships have been studied post mortem by Langewouters et al. [24] and described as mathematical functions of patients' age, sex, height and weight. Individual inaccuracy in aortic diameter determination translates into an inaccuracy in the absolute level of $\mathrm{CO}$ computed for an individual patient, but the ability to reliably track changes in $\mathrm{CO}$ remains intact. The uncertainty in total systemic peripheral resistance as a model parameter is removed as follows. For the first beat detected in the arterial pressure waveform, a population average value for peripheral resistance is assumed in the model and mean arterial pressure and $\mathrm{CO}$ are computed. The ratio of pressure to $\mathrm{CO}$ for this beat defines a new resistance used in the model for the next beats. Within 5 beats after the start, model resistance stabilizes to total systemic peripheral resistance. The model follows changes in resistance that further occur.

\section{Study protocol}

For each patient, the following data were collected: heart rate (HR), systolic arterial blood pressure $\left(P_{\text {sys }}\right)$, diastolic arterial blood pressure $\left(P_{\mathrm{dia}}\right)$, mean arterial blood pressure (MAP), mean pulmonary artery pressure (MPAP), central venous pressure (CVP), pulmonary artery occlusion pressure (PAOP; after proper wedging), $\mathrm{CO}$ by thermodilution and noncalibrated, modified ModelFlow (COtd, COmf, respectively), SVV, SPV and PPV, and their response to changes in PEEP and in $V_{t}$. Plateau and mean airway pressures and $V_{\mathrm{t}}$ were recorded. Blood pressures were taken after calibration and zeroing to atmospheric pressure, at the mid-chest level with patients in the supine position. CO was measured by means of the bolus thermodilution method, following triplicate central venous injections $\left(10 \mathrm{ml}\right.$ of glucose $5 \%$ at $\left.4-6^{\circ} \mathrm{C}\right)$, irrespective of the ventilatory cycle, and averaging. The noncalibrated, modified ModelFlow method was used to less invasively assess $\mathrm{CO}$ and SVV according to methods described earlier and explained above [21-23]. Radial artery pressure, taken from the monitor, was sampled by a computer system at $100 \mathrm{~Hz}$ and used as input to the model, to compute an aortic flow waveform. The flow waveform was integrated during arterial systole to deliver stroke volume. $\mathrm{CO}$ was computed for each beat as the product of stroke volume and HR. SVV was defined as the variation in beat-to-beat stroke volume during a single respiratory cycle and was calculated as: $\mathrm{SVV}=\left(\mathrm{SV}_{\max }-\mathrm{SV}_{\min }\right) / \mathrm{SV}_{\text {mean }}$. The algorithm uses a period of $30 \mathrm{~s}$ to calculate $S V_{\text {mean }}$. The time window was divided into four $7.5 \mathrm{~s}$ periods; for each $7.5 \mathrm{~s}$, the highest $\left(\mathrm{SV}_{\max }\right)$ and the lowest $\left(\mathrm{SV}_{\min }\right)$ values of stroke volume were determined and the average of the four $7.5 \mathrm{~s}$ intervals was used to calculate SVV. Similarly, SPV and $\mathrm{PPV}$ were calculated from the arterial pressure curve, from $\mathrm{SPV}=\left(\mathrm{SP}_{\max }-\mathrm{SP}_{\text {min }}\right) / \mathrm{SP}_{\text {mean }}$ and $\mathrm{PPV}=\left(\mathrm{PP}_{\max }-\mathrm{PP}_{\text {min }}\right) /$ $\mathrm{PP}_{\text {mean }}$, respectively. After baseline measurements at $5 \mathrm{cmH}_{2} \mathrm{O}$ PEEP, measurements were done after $5 \mathrm{cmH}_{2} \mathrm{O}$ increments to $15 \mathrm{cmH}_{2} \mathrm{O}$ of PEEP each $10 \mathrm{~min}$, followed by $10 \mathrm{~min}$ of baseline conditions and repeated measurements. Then, $V_{\mathrm{t}}$ was increased at $10 \mathrm{~min}$ intervals by 25 and $50 \%$, followed again by $10 \mathrm{~min}$ of baseline conditions and repeated measurements.

\section{Statistical analysis}

Data were distributed normally (Kolmogorov-Smirnov test). We calculated the mean of baseline values before and after the challenges, which did not statistically significantly differ, for comparison with the changes evoked by the ventilatory interventions using the paired $t$-test. Linear correlation coefficients were used to express relations. To compare COmf with COtd, Bland-Altman analysis was done plotting mean $\mathrm{CO}$ against the difference between COmf and COtd. Bias, precision (SD) and limits of agreement (bias $\pm 2 \mathrm{SD}$ ) were calculated. A $P$ value less than 0.05 was considered statistically significant and exact values are given if more than 0.001. Data are summarized by means $\pm S D$. 
Table 1 Patient characteristics $(n=17)$

\begin{tabular}{lc}
\hline M/F & $14 / 3$ \\
Height $(\mathrm{cm})$ & 175 \\
Weight $(\mathrm{kg})$ & 83 \\
Body surface area $\left(\mathrm{m}^{2}\right)$ & 2.01 \\
Type of surgery & $11 \mathrm{CABG}, 5 \mathrm{AVR}, 1 \mathrm{MVP}$ \\
EuroSCORE & $2 \pm 2$ \\
CPB time (min) & $100 \pm 53$ \\
Vasoactive drugs & \\
$\quad$ Dopamine $\left(\mu \mathrm{kg}^{-1} \mathrm{~min}^{-1}\right)$ & $5.5 \pm 3.5$ \\
$\quad$ Dobutamine $\left(\mu \mathrm{kg}^{-1} \mathrm{~min}^{-1}\right)$ & $5.0 \pm 3.6$ \\
$\quad$ Norepinephrine $\left(\mu \mathrm{gg}^{-1} \mathrm{~min}^{-1}\right)$ & $0.04 \pm 0.01$ \\
$\mathrm{P}_{\mathrm{a}} \mathrm{O}_{2} / \mathrm{F}_{1} \mathrm{O}_{2}$ & $311 \pm 91$ \\
\hline
\end{tabular}

Mean \pm SD. AVR, aortic valve replacement; CABG, coronary artery bypass graft; CPB; cardiopulmonary bypass; $\mathrm{F}$, female; $\mathrm{M}$, male; MVP, mitral valve repair; paO2/ fiO2, arterial $P O 2$ over inspiratory $\mathrm{O}_{2}$ fraction.

\section{Results}

Patients' characteristics are described in Table 1 and ventilatory changes during the protocol in Table 2. Plateau airway pressures similarly increased with PEEP and $V_{\mathrm{t}}$ increments. Table 3 shows that COmf decreased and SVV increased concomitantly with COtd decreases, as compared with baseline, by increasing PEEP. The change in COmf correlated with the change in mean airway pressure $(r=-0.46, P=0.011)$. Increasing $V_{\mathrm{t}}$ $\left(10 \pm 1 \mathrm{ml} \mathrm{kg}^{-1}\right.$ ) by 25 to $50 \%$ (to $15 \pm 1 \mathrm{ml} \mathrm{kg}^{-1}$ ) did not change SVV, SPV, PPV, COmf or COtd (Table 4). However, $P_{\text {sys }}, P_{\text {dia }}$, MAP, CVP and PAOP increased, without changing the PAOP-CVP gradient.

\section{Correlations with stroke volume variation}

COtd decreases were more than 10\% in eight of 34 steps during PEEP increments, and no variable, including SVV, was of predictive value at baseline. Changes in SVV did not relate to changes in COtd or COmf. However, for pooled data before, during and after PEEP, SVV inversely related to stroke volume by noncalibrated, modified ModelFlow $(r=-0.50, P=0.001)$ and not by thermodilution ( $r=0.38, P=0.796)$, but changes did not. For pooled data before, during and after PEEP increments, SPV and PPV related to SVV $(r=0.61$ and $r=0.74$, respectively, $P<0.001$ ), but changes did not.

\section{Comparing cardiac output by noncalibrated, modified ModelFlow with cardiac output by thermodilution}

For pooled data, that is, mean baseline values before, during and after PEEP and $V_{\mathrm{t}}$ increments, noncalibrated COmf correlated with COtd at an $r$ value of 0.52 and
$P$ value less than 0.001 (and stroke volumes at $r=0.65$, $P<0.001)$. Bland-Altman analysis revealed a bias between $\mathrm{CO}$ measurement techniques of $-0.361 \mathrm{~min}^{-1}$ (precision, $1.421 \mathrm{~min}^{-1}$ ) with limits of agreement between 2.47 and $-3.201 \mathrm{~min}^{-1}$. Figure 1 shows the relation between changes in COmf and COtd as a function of PEEP changes from 5 to 10 and from 10 to $15 \mathrm{cmH}_{2} \mathrm{O} \quad(r=0.55, \quad P=0.002)$. Changes in stroke volume measured by the techniques showed even better correlation $(r=0.77, P<0.001)$.

\section{Discussion}

In mechanically ventilated patients after cardiac surgery, a fall in less invasive (pulse-contour), noncalibrated and modified ModelFlow $\mathrm{CO}$ is more sensitive than the derived SVV, which is more sensitive than SPV and PPV, in tracking a fall in COtd during continuous (and not cyclic) increases in intrathoracic pressure.

In comparing $\mathrm{CO}$ measurement techniques, the limits of agreement of COmf with COtd (2SD/mean) were 47\%, well above the $30 \%$ criterion by Critchley and Critchley [25] for a theoretically acceptable alternative to thermodilution-derived CO. The data agree with our previous comparisons of noncalibrated, modified ModelFlow at steady state conditions after cardiac surgery [21,22]. Even though the noncalibrated, modified ModelFlow technique somewhat underestimated and not fully agreed with the thermodilution technique, it was apparently sensitive enough to detect decreases in thermodilution $\mathrm{CO}$ with continuous increases in intrathoracic pressure. Indeed, the accuracy of detecting rapid changes in $\mathrm{CO}$ may be clinically more useful than that of absolute numbers.

Baseline SVV, SPV and PPV were lower than often observed to be associated with preload (fluid) responsiveness [1-11,13-15], and baseline values did not predict the fall in CO during PEEP increments. Nevertheless, PEEP (and not $V_{\mathrm{t}}$ ) increments decreased $\mathrm{CO}$ and increased SVV and this can be explained as follows. Although increasing PEEP and mean airway pressure may mainly reduce venous return and thereby render CO preload-dependent, PEEP may also increase afterload of the right ventricle at inspiration, thereby contributing to a decrease in $\mathrm{CO}[16-$ 19]. Conversely, rises in $V_{\mathrm{t}}$ also increase lung volume and

Table 2 Ventilatory changes

\begin{tabular}{|c|c|c|c|c|c|c|}
\hline Baseline & PEEP10 & PEEP15 $\mathrm{cmH}_{2} \mathrm{O}$ & Baseline & $V_{t} 25 \%$ & $V_{t} 50 \%$ & Baseline \\
\hline \multicolumn{7}{|l|}{ PEEP, $\mathrm{cmH}_{2} \mathrm{O}$} \\
\hline & 10 & 15 & 5 & 5 & 5 & 5 \\
\hline \multicolumn{7}{|c|}{ Plateau airway pressure, $\mathrm{cmH}_{2} \mathrm{O}$} \\
\hline $25 \pm 4$ & $29 \pm 3^{*}$ & $32 \pm 3^{*}$ & $23 \pm 4$ & $29 \pm 5^{*}$ & $33 \pm 5^{*}$ & $25 \pm 4$ \\
\hline \multicolumn{7}{|c|}{ Mean airway pressure, $\mathrm{cmH}_{2} \mathrm{O}$} \\
\hline $9 \pm 1$ & $14 \pm 1^{*}$ & $18 \pm 1^{*}$ & $9 \pm 1$ & $10 \pm 1^{*}$ & $11 \pm 1^{*}$ & $9 \pm 1$ \\
\hline \multicolumn{7}{|l|}{ Tidal volume, $\mathrm{ml}$} \\
\hline $784 \pm 100$ & $784 \pm 100$ & $784 \pm 100$ & $784 \pm 100$ & $1003 \pm 133^{*}$ & $1202 \pm 174^{*}$ & $781 \pm 96$ \\
\hline
\end{tabular}

Means \pm SD. $V_{t} 25 \%$, tidal volume increased by $25 \% ; V_{t} 50$, tidal volume increased by $50 \%$. PEEP, positive end-expiratory pressure ${ }^{*} P<0.001$ versus mean of surrounding baseline values. 
Table 3 Haemodynamics and positive end-expiratory pressure

\begin{tabular}{|c|c|c|c|c|c|}
\hline & Mean baseline & PEEP10 $\mathrm{cmH}_{2} \mathrm{O}$ & $P$ & PEEP15 $\mathrm{cmH}_{2} \mathrm{O}$ & $P$ \\
\hline HR (beats $\min ^{-1}$ ) & $80 \pm 12$ & $80 \pm 14$ & 0.854 & $79 \pm 13$ & 0.250 \\
\hline$P_{\text {sys }}(\mathrm{mmHg})$ & $125 \pm 13$ & $124 \pm 16$ & 0.545 & $116 \pm 18$ & 0.022 \\
\hline$P_{\text {dia }}(\mathrm{mmHg})$ & $67 \pm 9$ & $68 \pm 11$ & 0.722 & $64 \pm 10$ & 0.153 \\
\hline MAP (mmHg) & $87 \pm 11$ & $85 \pm 13$ & 0.190 & $82 \pm 13$ & 0.056 \\
\hline MPAP $(\mathrm{mmHg})$ & $21 \pm 6$ & $23 \pm 7$ & 0.007 & $24 \pm 5$ & $<0.001$ \\
\hline PAOP (mmHg) & $10 \pm 5$ & $11 \pm 5$ & 0.015 & $13 \pm 5$ & $<0.001$ \\
\hline CVP $(\mathrm{mmHg})$ & $9 \pm 4$ & $10 \pm 4$ & 0.056 & $11 \pm 3$ & 0.004 \\
\hline COtd $\left(I \min ^{-1}\right)$ & $5.7 \pm 1.2$ & $5.6 \pm 1.5$ & 0.250 & $5.1 \pm 1.2$ & $<0.001$ \\
\hline $\operatorname{COmf}\left(I \min ^{-1}\right)$ & $6.2 \pm 1.3$ & $6.0 \pm 1.4$ & 0.017 & $5.5 \pm 1.3$ & $<0.001$ \\
\hline SVV (\%) & $6.2 \pm 4.4$ & $8.1 \pm 5.6$ & 0.064 & $7.8 \pm 3.5$ & 0.031 \\
\hline SPV (\%) & $6.0 \pm 2.5$ & $5.8 \pm 3.0$ & 0.535 & $6.9 \pm 3.5$ & 0.284 \\
\hline PPV (\%) & $6.4 \pm 4.3$ & $6.6 \pm 4.0$ & 0.788 & $7.0 \pm 3.4$ & 0.593 \\
\hline
\end{tabular}

Means \pm SD. COmf, cardiac output by noncalibrated, modified ModelFlow; COtd, cardiac output thermodilution; CVP, central venous pressure; HR, heart rate; MAP, mean arterial blood pressure; MPAP, mean pulmonary artery pressure; $\mathrm{PAOP}$, pulmonary artery occlusion pressure; $\mathrm{PEEP} 10$, positive end-expiratory pressure of 10 cm $\mathrm{H}_{2} \mathrm{O}$; PEEP15, PEEP $15 \mathrm{cmH}_{2} \mathrm{O} ; P_{\text {dia }}$ diastolic blood pressure; $P_{\text {sys }}$, systolic blood pressure; PPV, pulse pressure variation; SPV, systolic pressure variation; SVV, stroke volume variation.

thereby afterload of the right ventricle at inspiration, but apparently did not raise mean airway pressure to levels with PEEP and therefore did not affect preload-dependent $\mathrm{CO}$ or SVV in our study. Conversely, right ventricular afterload changes may have played only a minor role in the PEEPinduced circulatory changes, which can therefore be mainly attributed to mean airway pressure-related falls in right and, via series interaction, in left ventricular preload, in agreement with prior suggestions $[14,16,18]$. Apparently, the fall in preload decreased stroke volume and $\mathrm{CO}$ by the left ventricle and rendered it more sensitive to mechanical ventilation-induced variations in preload, thereby increasing SVV, as in animals [18]. In contrast, authors observed an increase in dynamic indices with increases in $V_{\mathrm{t}}$ $[8,12,13,15]$. The discrepancy with our results can be explained, in part, by lower preload at baseline in their studies than in ours. Moreover, increasing $V_{\mathrm{t}}$ somewhat increased arterial blood pressure, perhaps by sympathetic activation and resultant peripheral vasoconstriction, and thereby increased left ventricular afterload, whereas CO was maintained by concomitantly increased filling pressures. The unaltered gradient between CVP and PAOP may be explained if increased $V_{\mathrm{t}}$ augmented both right and left ventricular afterload as well as preload, as described previously [19]. Indeed, SVV may not be affected by after- loading of the left ventricle, if adequately filled [20]. We did not assess the interaction between PEEP and $V_{\mathrm{t}}$ on $\mathrm{SVV}$, for safety reasons, so that it cannot be excluded that SVV was more sensitive to $\mathrm{CO}$ changes during incremental PEEP at $V_{\mathrm{t}}$ more than $10 \mathrm{ml} \mathrm{kg}^{-1}$ than at lower $V_{\mathrm{t}}$.

The observation that SVV was more affected by a fall in CO with PEEP than the SPV and PPV can be explained, among other reasons, by pressure variations that are only partly caused by SVV [19]. Indeed, SPV and PPV proved more sensitive than SVV in reflecting preload dependency and fluid responsiveness of $\mathrm{CO}$ after cardiac surgery in previous studies, in which patients may have been underfilled [2,13]. In contrast, our data suggest that PPV and SPV are too insensitive to detect relatively small $\mathrm{CO}$ changes upon PEEP increases in (probably adequately filled) patients. Indeed, the SVV, SPV as well as the PPV were less helpful in monitoring decreases in COtd by changes in ventilator settings than the less invasive, noncalibrated COmf itself. Many pulse-contour methods have been evaluated [3,22], but, unlike the noncalibrated, modified ModelFlow technique, most of them require calibration by an independent and invasive CO measurement and only a few have been evaluated for their ability to track preload (fluid) responsiveness or responses to ventilatory changes [26].

Table 4 Haemodynamics and tidal volume

\begin{tabular}{|c|c|c|c|c|c|}
\hline & Mean baseline & $V_{\mathrm{t}} 25 \% \mathrm{ml}$ & $P$ & $V_{\mathrm{t}} 50 \% \mathrm{ml}$ & $P$ \\
\hline HR (beats $\min ^{-1}$ ) & $79 \pm 13$ & $80 \pm 12$ & 0.031 & $80 \pm 12$ & 0.171 \\
\hline$P_{\text {sys }}(\mathrm{mmHg})$ & $125 \pm 15$ & $125 \pm 16$ & 0.495 & $128 \pm 15$ & 0.046 \\
\hline$P_{\text {dia }}(\mathrm{mmHg})$ & $67 \pm 9$ & $67 \pm 9$ & 0.971 & $69 \pm 9$ & 0.003 \\
\hline MAP (mmHg) & $88 \pm 11$ & $88 \pm 12$ & 0.903 & $90 \pm 11$ & 0.008 \\
\hline MPAP $(\mathrm{mmHg})$ & $21 \pm 6$ & $21 \pm 5$ & 0.319 & $21 \pm 6$ & 0.754 \\
\hline PAOP $(\mathrm{mmHg})$ & $10 \pm 4$ & $11 \pm 4$ & 0.110 & $11 \pm 5$ & 0.054 \\
\hline CVP $(\mathrm{mmHg})$ & $9 \pm 4$ & $10 \pm 4$ & 0.633 & $10 \pm 4$ & 0.005 \\
\hline $\operatorname{COtd}\left(I \min ^{-1}\right)$ & $5.9 \pm 1.5$ & $6.0 \pm 1.5$ & 0.675 & $5.9 \pm 1.7$ & 0.340 \\
\hline $\operatorname{COmf}\left(\operatorname{lmin}^{-1}\right)$ & $6.3 \pm 1.5$ & $6.3 \pm 1.5$ & 0.136 & $6.3 \pm 1.6$ & 0.234 \\
\hline SVV (\%) & $5.2 \pm 3.1$ & $6.8 \pm 2.9$ & 0.073 & $6.3 \pm 2.3$ & 0.249 \\
\hline SPV (\%) & $6.2 \pm 2.8$ & $6.1 \pm 3.5$ & 0.952 & $6.9 \pm 3.4$ & 0.471 \\
\hline PPV (\%) & $6.3 \pm 4.3$ & $5.6 \pm 3.3$ & 0.517 & $7.1 \pm 3.3$ & 0.568 \\
\hline
\end{tabular}

Means \pm SD. $V_{t} 25 \%$, tidal volume increased by $25 \% ; V_{t} 50 \%$, tidal volume increased by $50 \%$. COmf, cardiac output by noncalibrated, modified ModelFlow; COtd, cardiac output thermodilution; CVP, central venous pressure; HR, heart rate; MAP, mean arterial blood pressure; MPAP, mean pulmonary artery pressure; PAOP, pulmonary artery occlusion pressure; $P_{\text {dia, }}$ diastolic blood pressure; $P_{\text {sys }}$, systolic blood pressure; PPV, pulse pressure variation; SPV, systolic pressure variation; SVV, stroke volume variation. 
Fig. 1

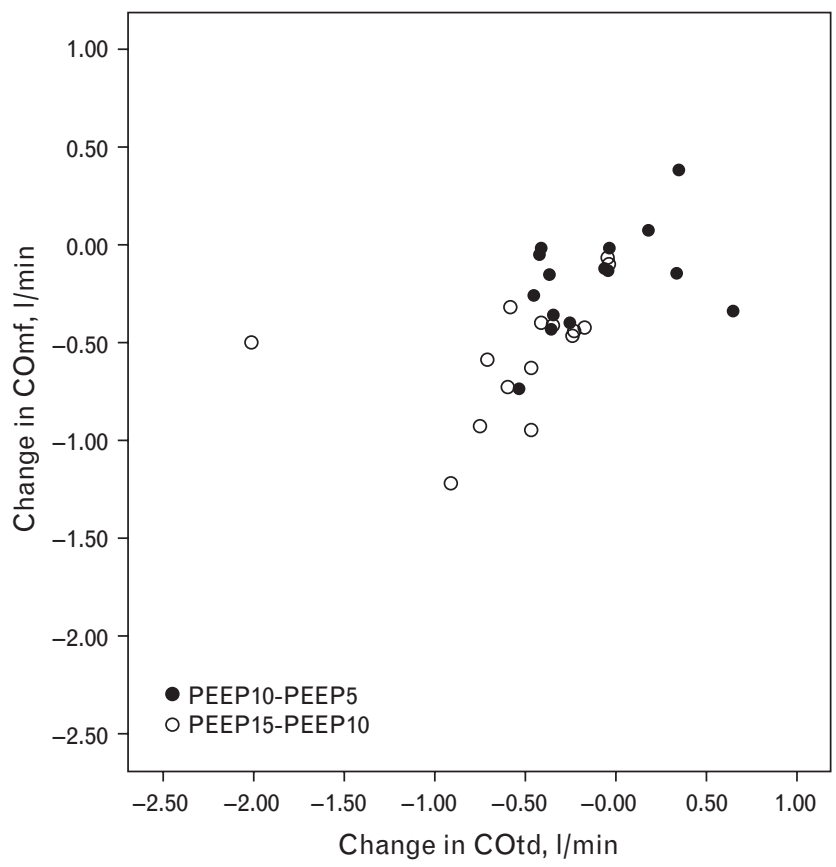

Changes in less invasive, noncalibrated, modified ModelFlow cardiac output (COmf, I $\mathrm{min}^{-1}$ ) as a function of positive end-expiratory pressure (PEEP) increments versus changes in thermodilution cardiac output $\left(\mathrm{COtd}, \mid \mathrm{min}^{-1}\right): r=0.55, P=0.002$.

In conclusion, a fall in noncalibrated, modified ModelFlow CO is more sensitive than a rise in SVV, which is more sensitive than SPV and PPV, in tracking a fall in COtd during continuous (and not cyclic) increases in intrathoracic pressure, in mechanically ventilated, adequately filled patients after cardiac surgery. This suggests a reduction in biventricular preload as the main factor in decreasing $\mathrm{CO}$ and, as a result, increasing SVV with PEEP. The findings may help to understand pulse-contour monitoring and underscore the value of noncalibrated, modified ModelFlow to track circulatory changes during changes in ventilatory settings.

\section{References}

1 Reuter DA, Felbinger TW, Schmidt C, et al. Stroke volume variations for assessment of cardiac responsiveness to volume loading in mechanically ventilated patients after cardiac surgery. Intensive Care Med 2002; 28:392398.

2 Reuter DA, Kirchner A, Felbinger TW, et al. Usefulness of left ventricular stroke volume variation to assess fluid responsiveness in patients with reduced cardiac function. Crit Care Med 2003; 31:1399-1404.

3 Kramer A, Zygun D, Hawes $\mathrm{H}$, et al. Pulse pressure variation predicts fluid responsiveness following coronary artery bypass surgery. Chest 2004 ; 126:1563-1568.

4 Rex S, Brose S, Metzelder S, et al. Prediction of fluid responsiveness in patients during cardiac surgery. Br J Anaesth 2004; 93:782-788.

5 De Backer D, Heenen S, Piagnerelli M, et al. Pulse pressure variations to predict fluid responsiveness: influence of tidal volume. Intensive Care Med 2005; 31:517-523.

6 Hofer CK, Müller SM, Furrer L, Klaghofer R. Stroke volume and pulse pressure variation for prediction of fluid responsiveness in patients undergoing off-pump coronary artery bypass grafting. Chest 2005 128:848-854.
7 Preisman S, Kogan S, Berkenstadt H, Perel A. Predicting fluid responsiveness in patients undergoing cardiac surgery: functional haemodynamic parameters including the respiratory systolic variation test and static preload indicators. $\mathrm{Br} J$ Anaesth 2005; 95:746-755.

8 Wiesenack C, Fiegl C, Keyser A, et al. Assessment of fluid responsiveness in mechanically ventilated cardiac surgical patients. Eur J Anaesthesiol 2005; 22:658-665.

9 Rex S, Schälte G, Schroth S, et al. Limitations of arterial pulse pressure variation and left ventricular stroke volume variation in estimating cardiac preload during open heart surgery. Acta Anaesthesiol Scand 2007; 51:1258-1267.

10 Auler JO, Galas F, Hajjar L, et al. Online monitoring of pulse pressure variation to guide fluid therapy after cardiac surgery. Anesth Analg 2008; 106:1201-1206.

11 Michard F, Chemla D, Richard C, et al. Clinical use of respiratory changes in arterial pulse pressure to monitor the hemodynamic effects of PEEP. Am $J$ Respir Crit Care Med 1999; 159:935-939.

12 Reuter DA, Felbinger TW, Kilger E, et al. Optimizing fluid therapy in mechanically ventilated patients after cardiac surgery by on-line monitoring of left ventricular stroke volume variations. Comparison with aortic systolic pressure variations. Br J Anaesth 2002; 88:124-126.

13 Reuter DA, Bayerlein J, Goepfert MS, et al. Influence of tidal volume on left ventricular stroke volume variation measured by pulse contour analysis in mechanically ventilated patients. Intensive Care Med 2003; 29:476480.

14 Charron C, Fessenemeyer C, Cosson C, et al. The influence of tidal volume on the dynamic variables of fluid responsiveness in critically ill patients. Anesth Analg 2006; 102:1511-1517.

15 De Blasi RA, Palmisani S, Cigognetti L, et al. Effects of sternotomy on heartlung interaction in patients undergoing cardiac surgery receiving pressurecontrolled mechanical ventilation. Acta Anaesthesiol Scand 2007; 51:441 446.

16 Luecke T, Pelosi P. Clinical review: positive end-expiratory pressure and cardiac output. Crit Care 2005; 9:607-621.

17 Pizov R, Cohen M, Weiss Y, et al. Positive end-expiratory pressure-induced hemodynamic changes are reflected in the arterial pressure waveform. Crit Care Med 1996; 24:1381-1387.

18 Kubitz JC, Annecke T, Kemming Gl, et al. The influence of positive endexpiratory pressure on stroke volume variation and central blood volume during open and closed chest conditions. Eur J Cardiothorac Surg 2006; 30:90-95.

19 Veillard-Baron A, Chergui K, Augarde R, et al. Cyclic changes in arterial pulse during respiratory support revisited by Doppler echocardiography. Am J Respir Crit Care Med 2003; 168:671-676.

20 Kubitz JC, Annecke T, Forkl S, et al. Validation of pulse contour derived stroke volume variation during modifications of cardiac afterload. $\mathrm{Br} J$ Anaesth 2007; 98:591-597.

21 De Vaal JB, de Wilde RB, van den Berg PCM, et al. Less invasive determination of cardiac output from the arterial pressure by aortic diameter-calibrated pulse contour. $\mathrm{Br} J$ Anaesth 2005; 95:326331.

22 De Wilde RB, Schreuder JJ, van den Berg PC, Jansen JR. An evaluation of cardiac output by five arterial pulse contour techniques during cardiac surgery. Anaesthesia 2007; 62:760-768.

23 Jellema WT, Wesseling KH, Groeneveld AB, et al. Continuous cardiac output in septic shock by simulating a model of the aortic input impedance: a comparison with bolus injection thermodilution. Anesthesiology 1999; 90:1317-1328.

24 Langewouters GJ, Wesseling KH, Goedhard WJ. The static elastic properties of 45 human thoracic and 20 abdominal aortas in vitro and the parameters of a new model. J Biomech $1984 ; 17: 425-435$.

25 Critchley LAH, Critchley JAJH. A meta-analysis of studies using bias and precision statistics to compare cardiac output measurement techniques. J Clin Monitor Comput 1999; 15:85-91.

26 Felbinger TW, Reuter DA, Eltzschig HK, et al. Cardiac index measurements during rapid preload changes: a comparison of pulmonary artery thermodilution with arterial pulse contour analysis. J Clin Anesth 2005; 17:241-248. 